\title{
ARTIGOS
}

\section{LA SOCIOLOGÍA COMO PROFESIÓN: DESENCUENTROS ENTRE LA FORMACIÓN ACADÉMICA Y LA INSERCIÓN LABORAL ${ }^{1,2}$}

MARÍA AGUSTINA DIEZ

\section{RESUMEN}

Varios autores han señalado la tensión existente entre la formación universitaria de ciertas disciplinas y la inserción profesional en el mercado de trabajo en general, y en particular para el caso de la sociología. El presente trabajo tiene como objetivo principal analizar la forma en que los sociólogos egresados de la Universidad Nacional de Cuyo -UNCuyo- han ido construyendo sus carreras profesionales, la relación entre el tipo de formación recibida, sus percepciones, sus prácticas laborales y las alternativas existentes de inserción laboral a las que deben enfrentarse en la actualidad. Se trata de un estudio exploratorio caracterizado por un abordaje de tipo cualitativo. Se trabajó a través de la realización de entrevistas biográficas a graduados de la carrera, pertenecientes a distintas cohortes.

\section{ENSEÑANZA SUPERIOR • FORMACIÓN PROFESIONAL・EMPLEO・ MERCADO LABORAL}

\section{SOCIOLOGY AS A PROFESSION: GAPS BETWEEN ACADEMIC EDUCATION AND LABOR INSERTION}

\section{ABSTRACT}

Several authors have highlighted the tension between university education in certain disciplines and professional insertion in the job market in general, especially regarding sociology. This study aims to analyze the way in which sociologist graduates from Universidad Nacional de Cuyo - UNCuyo -, were building their professional careers, as well as the relationship between the type of education received, their perceptions, labour practices and existing alternatives of labour insertion which they must face today. This is an exploratory study characterized by a qualitative approach. The study was carried out through biographical interviews with sociologists from several cohorts. 
Plusieurs auteurs ont mis en relief la tension entre la formation universitaire et l'insertion professionnelle dans le marché du travail. C'est particulièrement, le cas de la sociologie. L'objectif principal de cette étude est d'analyser la manière dont les sociologues issus de l'Universidad Nacional de Cuyo-UNCuyo - ont construit leurs carrières professionnelles, le rapport entre le type de formation reçue et leurs perceptions et pratiques de travail, ainsi que les alternatives d'insertion dans le monde du travail auxquelles ils sont confrontés aujourd'hui. Il s'agit d'une étude exploratoire, caractérisée par une approche qualitative. L'enquête par moyen d'entretiens biographiques a été menée aupres de diplômés en sociologie, provenant de différentes cohortes.

ENSEIGNEMENT SUPÉRIEUR • FORMATION PROFESSIONNELLE • EMPLOI •

MARCHÉ DU TRAVAIL

\section{A SOCIOLOGIA COMO PROFISSÃO: DESENCONTROS ENTRE A FORMAÇÃO ACADÊMICA E A INSERÇÃO LABORAL}

RESUMO

Vários autores destacaram a tensão existente entre a formação universitária de certas disciplinas e a inserção profissional no mercado de trabalho em geral, sobretudo no caso da sociologia. O presente trabalho tem o objetivo principal de analisar a forma em que os sociólogos formados na Universidad Nacional de Cuyo - UNCuyo - foram construindo suas carreiras profissionais, bem como a relação entre o tipo de formação recebida, suas percepções, práticas trabalhistas $e$ as alternativas existentes de inserção laboral que devem enfrentar atualmente. Trata-se de um estudo exploratório caracterizado por uma abordagem qualitativa. o trabalho foi realizado por meio de entrevistas biográficas com sociólogos pertencentes a diversas coortes. 


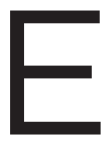

xiste cierto consenso al afirmar que a partir de los años 80, con el advenimiento de la democracia, se pudo observar una notable expansión de los espacios de inserción profesional que fueron ocupando los sociólogos (TESTA, 1996; BELTRÁN, 2005; RUBINICH; BELTRÁN, 2010; BLOIS, 2011, 2012, 2014; PEREYRA et al., 2015). Esta expansión implicaba no solo crecimiento sino una diversificación y redefinición de los lugares ocupados por estos profesionales:

BIZAI, Jorgelina; STECHINA Mariana. La inserción de los sociólogos en el ámbito universitario. Buenos Aires, 2003. mimeo.

GARCIA, Bibiana. El mundo de las consultoras. Un ámbito en crecimiento de la sociología argentina en la década del '90". Buenos Aires, 2003. mimeo.

EECARIA, Alejand LLDFARB, Lucía. El campo de la sociología a partir del

estudio de la inserción de los sociólogos en el Estado. Buenos Aires, 2003. mimeo.
Así, aun cuando el académico continuó siendo el espacio privilegiado en términos de reconocimiento (Bizai y Stechina, $2003^{3}$ ), otros espacios como la consultoría en opinión pública y estudios de mercado (García, 2003), la función pública (Beccaria y Goldfarb, $2003^{5}$ ) y la gran empresa privada (Casco y Engelman, 20036) aumentaron considerablemente la demanda de sociólogos, transformando el campo de la Sociología. (BELTRÁN, 2005, p. 485)

Algunas de las razones fundamentales de la expansión de los espacios de inserción aducidas han sido las aceleradas transformaciones que comenzó a atravesar la sociedad argentina en los 90, junto con los procesos de creciente racionalización estatal y de la empresa privada. Todos estos procesos implicaron entonces "una profunda redefinición del mapa de la sociología local” (BLOIS, 2011, p. 197). Lógicamente estas transformaciones han presentado más interrogantes que certezas: ¿en 
qué consisten las prácticas profesionales de los sociólogos actualmente? ¿Cómo son sus recorridos laborales? ¿Qué concepción tienen los sociólogos sobre las posibilidades laborales que les ha brindado la formación de grado? Y sobre todo, ¿qué particularidades reviste la situación a nivel local?

El presente trabajo tiene como objetivo principal analizar cómo los sociólogos egresados de la Universidad Nacional de Cuyo -UNCuyo-, han ido trazando sus carreras profesionales, la relación entre el tipo de formación recibida, sus percepciones, sus prácticas laborales y las alternativas existentes de inserción laboral a las que deben enfrentarse en la actualidad. Esta investigación pretende situarse dentro del fértil terreno de los estudios de formación y empleo, generados a partir del cruce entre la sociología del trabajo y la sociología de las profesiones (PANAIA, 2008, 2009).

A lo largo de este análisis intentaremos observar, desde la percepción de los propios sociólogos, qué grado de articulación existe entre el plan de estudios de la carrera, ${ }^{7}$ el proceso de formación, la socialización académica y las alternativas reales de inserción laboral para estos profesionales.

Se trata de un estudio exploratorio caracterizado por un abordaje de tipo cualitativo. Se trabajó a través de la realización de 25 entrevistas biográficas (MUÑIZTERRA, 2012) a graduados de la carrera, pertenecientes a las cohortes que van de 1983 al año $1999^{8}$ y de la construcción de tablas de empleo para cada uno de ellos, además del análisis de sus currículos vitae. En este primer análisis, los datos obtenidos se han utilizado en función de captar la diversidad de situaciones y de intentar encontrar algunos elementos que aporten a una visión global del tema y en un próximo trabajo serán utilizados para interpretar cada trayectoria en toda su profundidad.

Partimos entendiendo las trayectorias laborales como el producto de un complejo de factores cuyos antecedentes no comienzan con los primeros trabajos sino que se elaboran antes y por la acción de varios elementos (LONGO, 2011). Las características de la historia previa van dando forma a las experiencias y a las prácticas laborales. Dichas características son una parte de lo que denominaremos el proceso de socialización profesional.

Augusto de Venanzi (1990) propone un modelo para la compresión de los procesos de socialización profesional, que nos ha servido como punto de partida para el análisis, pero que sin embargo hemos necesitado ampliar y reformular. Este modelo está dividido en tres fases: la presocialización, que concierne principalmente a aquellas actitudes ocupacionales a que está expuesto el sujeto antes de ingresar a la escuela universitaria y que responden, entre otras cosas, a las opiniones que sus familiares y amigos expresan sobre determinadas carreras. ${ }^{9}$ Luego

7

El Ministerio de Educación de la Nación, en su Resolución n540/89, reconocía las incumbencias del título de Licenciado en Sociología otorgado por la Universidad Nacional de Cuyo. Dichas incumbencias son las mismas que ese Ministerio estableció en el

año 1986 para todas las carreras de Sociología del país (Resolución n 1818/86) 8

Se entiende como "cohorte" el conjunto de sujetos que ingresaron a la carrera en un determinado año y que obtuvieron el título, sin importar el año en el que egresaron de la misma.

\section{9}

Las dimensiones

relacionadas con esta etapa y trabajadas en las entrevistas fueron: características de la familia de origen, el colegio al que asistieron, las actividades extraescolares que realizaron, y la percepción de los entrevistados sobre: los factores, los motivos y las personas que influyeron en su decisión. 
la socialización académica, la cual está asociada a las actitudes y valores ocupacionales que el sujeto reconoce en sus profesores universitarios. Y en este caso deberíamos agregar también el conjunto de conocimientos, experiencias y prácticas internalizadas durante la etapa del cursado que no se ciñe exclusivamente al cursado de las materias. ${ }^{10}$

Finalmente, en el ingreso a las diferentes organizaciones o espacios laborales, los sujetos entran en contacto con nuevos marcos normativos constituidos por las ideologías, valores y subculturas predominantes en dichos espacios que también incidirán fuertemente en sus trayectorias. Esta etapa es denominada "socialización profesional". Como señala De Venanzi, "el ingreso de los profesionales al mundo del trabajo plantea serios retos a la supervivencia de los valores adquiridos durante la socialización académica” (1990, p. 140). En otras palabras, si nuestro objetivo es intentar comprender las relaciones sociales que los sujetos entablan en el mundo del trabajo, las decisiones tomadas, los cambios de rumbo o la permanencia en determinados empleos, es necesario tener en cuenta las características propias de cada etapa en el proceso de socialización profesional.

Para poder realizar el análisis de la trayectoria laboral propiamente dicha, nos enfocamos en las siguientes dimensiones: primer trabajo, trabajos durante los estudios universitarios, trabajos luego de recibirse y finalmente, trabajo actual. Además, indagamos sobre la percepción que tienen sobre sí mismos y su profesión, la situación del mercado de trabajo, los proyectos a futuro y la relación con otras profesiones.

\section{LA EXPERIENCIA DE SER ESTUDIANTE DE SOCIOLOGÍA}

El total de graduados que ingresaron entre 1983 y 1999 es de 337. El 72\% son mujeres y el $28 \%$ restante son hombres. Se realizaron 25 entrevistas biográficas a graduados de la carrera (17 Mujeres y 8 Hombres), pertenecientes a las dichas cohortes. Los entrevistados nacieron entre 1961 y 1980, es decir que tienen entre 36 y 55 años. Y al menos cuentan con 5 años de experiencia laboral luego de haber obtenido el título. La gran mayoría de los entrevistados proviene de familia de sectores medios.

Como señalamos más arriba, la experiencia como estudiantes universitarios, el conjunto de conocimientos y destrezas disciplinares, las normas, valores y representaciones adquiridas en esos años van a contribuir fuertemente en la conformación de la trayectoria del futuro sociólogo/a. Durante el cursado van instalándose ciertas concepciones sobre lo que es y lo que no es un "buen sociólogo" a través de lo que se constituye como las principales tradiciones sociológicas y corrientes intelectuales que marcan cómo debe encararse dicho oficio (WRIGHT MILLS, 1959; BOURDIEU, 2002, por citar algunos ejemplos). El ámbito 
académico es visto como el espacio por excelencia para ejercer la profesión, entonces "ser investigador" o "ser docente en la universidad" se convierte en la cara visible de la futura inserción profesional prestigiosa.

Sumado a esto, se encuentra la imagen del sociólogo como intelectual militante. Sobre todo en las primeras cohortes analizadas (aquellas correspondientes a los primeros años posteriores al inicio de la democracia) se observa una composición muy heterogénea con respecto a la edad. En esos años se produjo el ingreso de una cantidad importante de estudiantes de entre 30 y 50 años con una previa experiencia militante en distintos partidos y otras organizaciones. Esto puede considerarse como uno de los factores que reforzó en la carrera la idea del sociólogo como intelectual comprometido. Por supuesto no podemos dejar de considerar que la sociología desde sus orígenes como carrera universitaria ha estado muy unida a la militancia política, más que muchas otras disciplinas (DELICH, 1977; BLANCO, 2006; RUBINICH, 1999; NOÉ, 2005; PEREYRA, 2007; GONZALEZ, 2000; DIEZ, 2009, 2012). Y en este sentido la carrera de sociología de la UNCuyo no escapa a esa caracterización. La fuerte presencia de la militancia estudiantil durante la etapa de cursado marca también su impronta sobre de lo que se supone que es un "sociólogo comprometido" y qué trabajos estarían o no acordes con ese ideal.

Desde esta perspectiva, resulta difícil entonces pensar otras alternativas de inserción, como por ejemplo el trabajar en el Estado: “en el (ámbito) público yo no quería trabajar. Cuando empecé a estudiar yo dije lo único que yo no quiero ser es ser socióloga del Estado" (socióloga, 45 años, recibida en 1998, sector estatal). Se percibe que trabajar en el Estado es "como venderse":

\footnotetext{
Una idea bastante romántica, porque al mismo tiempo vos entendías que las políticas sociales, todas las políticas públicas, tenían que tener intervención de un sociólogo, para poder ordenarse cualquiera, hasta rentas, cualquiera, o sobre todo rentas te diría [risas]. Y al mismo tiempo nadie quería trabajar para el Estado, porque trabajar para el Estado era, y eso pasa actualmente, no quieren trabajar para el Estado porque trabajar para el Estado es como venderse. (sociólogo, 55 años, graduado en 1991, sector privado)
}

Durante la etapa de cursado se fue imponiendo también una concepción del sociólogo contrapuesta al del trabajador social, quien sería el que interviene en la práctica. Por otro lado, los referentes más cercanos que ellos tuvieron en la carrera fueron los propios profesores que a su vez, en la mayoría de los casos, solo se dedicaban a la actividad académica. Y esto terminó reforzando esa idea. 
La posibilidad de trabajar en el ámbito privado también producía cierto rechazo en algunos: "En la facultad, lo privado era mala palabra" (sociólogo, 51 años recibido en 1991, sector académico).

\begin{abstract}
A mi nunca me gustó el tema de, bueno, hacer encuestas, o consultoras que era otro tema que se veía muy marcado en ese momento, sobre todo en mis amigos o sea listo pegar laburo en una consultora, seguir haciendo el caminito, hacer encuestas, encuestas hasta que después pasar a supervisar, ser supervisor de encuestas y después trabajar en la consultora, y la verdad que ese caminito privado no me interesaba en lo más mínimo. (sociólogo, 38 años, graduado en 2008, sector estatal y docente en media)
\end{abstract}

Aparece entonces, un sentimiento confuso entre lo que se quiere o espera realizar y al servicio de quiénes se espera poder trabajar una vez recibidos, y aquellas actividades que efectivamente puedan generar un ingreso que permita vivir de la profesión:

Esa percepción estaba, entonces generaba cierta confusión en el sentido que vos ibas a trabajar sobre todo de apoyo a organizaciones sociales, que claro, si la pensabas un poquito no te lo iban a poder pagar [risas]. Claro, el tema para vivir era medio complicado. (sociólogo, 55 años, recibido en 1991, sector privado)

Otro ejemplo de las nociones impartidas durante el proceso de socialización académica:

[...] porque en realidad me parece que tenía que ver con los lineamientos de la facultad, con lo que te decían cuando vos ingresabas, bueno, si vos vas a hacer sociología. Yo siempre me acuerdo que algún profesor nos dijo: bueno, ustedes van a ser o encuestadores o van a generar teoría, y yo decía iah bueno... La verdad es que yo decía, bueno, no quiero ninguna de las dos cosas. (socióloga, 53 años, recibida en 1997, sector académico)

Al llegar a los últimos años de la carrera, aparece una fuerte sensación de incertidumbre no solo a nivel individual sino entre los propios compañeros también:

Pero yo estaba fascinada con las materias... Si me pasó, cuando estaba en cuarto año, un día con mis compañeras, salimos en un recreo y dijimos "Che... ¿y qué hace un sociólogo?" [Risas]. Porque claro, cuando nos... Yo te digo que a mí, hoy en día, por ahi me cuesta explicar lo que hacemos. Este... fue como decir "bueno..." 
Claro porque, dice una "a mí me preguntan ¿qué hace un sociólogo? Y yo no sé qué contestar". Porque claro, vos decís "¿qué hace un médico?" Todo el mundo sabe lo que hace un médico, no te lo preguntan. Pero "¿qué hace un sociólogo?" Fue... Como decir "me estoy por recibir y no sé qué carajo voy a hacer. Voy a trabajar..." Eso fue como... Como... Dura esa parte, darme cuenta, digamos, que ni yo misma sabía qué iba hacer. Eso fue, como fuerte. iEstábamos todas en la misma situación! Porque fue el comentario generalizado. (socióloga, 40 años, recibida en 2003, sector estatal)

Durante la etapa del cursado no se reflexionaba, ya sea en forma individual o colectiva, sobre cuál era el perfil profesional del sociólogo. Es decir, se hablaba poco de lo que se podía hacer después de recibido. Usualmente, esta preocupación surgía en el último año del cursado, sin embargo, aún en ese momento tampoco se tenía la sensación de entender claramente qué alternativas concretas existían:

\begin{abstract}
[...] entonces como que no le veías una salida real, concreta y ahi veías como avanzaban mis compañeros que estaban estudiando otras carreras que ya tenían algo más...entonces tenías una crisis importante de qué iba a ser el resto de mi vida con respecto al trabajo. (sociólogo, 38 años, graduado en 2008, sector estatal y docente en media)
\end{abstract}

En muchas de estas trayectorias se observa que comienza a dilatarse el momento del egreso. ${ }^{11}$ Claramente esto se debe a muchos factores. Si bien es cierto que la elaboración de la tesina conlleva ciertas dificultades dado que se trata prácticamente del primer trabajo de investigación de mayor envergadura que realizan en forma individual, ésta no es la única razón para explicar el retraso del egreso. Una de las razones es que algunos ya empiezan a buscar trabajos con horarios fijos e ingresos estables lo cual les impide dedicarle tiempo a la elaboración de la misma. Y otra de las razones es, justamente, el sentir que se está acercando el momento de recibirse y la incertidumbre experimentada por la futura inserción laboral:

[...] (mirando) Para atrás, (veo) que me costó mucho también
decir bueno me recibo y ahora qué, qué hago, qué soy, para qué
me formé, hacia dónde voy, creo que eso también me llevó a
demorarme en hacer la tesis. (socióloga, 40 años, recibida en 2001,
sector estatal-privado) 11

En este sentido, es importante aclarar que en los últimos años se viene implementando en la carrera un plan denominado "futuros egresados" que intenta apoyar a los alumnos de $4^{\circ}$ y $5^{\circ}$ que han tenido dificultades y se han retrasado para recibirse. 


\section{LA FORMACIÓN DE GRADO EN SOCIOLOGÍA}

La formación teórica es percibida como sólida. Posibilita tener una mirada global y compleja sobre la realidad, una capacidad de análisis de las situaciones que se generan en sus trabajos y que ellos mismos ven que otros profesionales no tienen. Si bien en cada caso particular se mencionaron algunas falencias y debilidades en la formación teórica en relación a determinadas asignaturas, en general la misma es percibida como una especie de "brújula" que les ha permitido orientarse en los casos en donde sus trabajos han implicado la necesidad de profundizar algún aspecto en particular en forma autodidacta.

En otras palabras, la definen como una formación amplia que otorga insumos generales y que permite orientar la trayectoria hacia cualquier problemática particular, brindando la capacidad de producir visiones alternativas sobre las problemáticas sociales y aportar eso en el trabajo en equipo. "Es una formación que permite ser capaz de captar el tipo de sujeto con el que a uno le toca trabajar, sobre todo en el caso de poblaciones más vulnerables" (sociólogo, 43 años, recibido en 2004, sector estatal y docente nivel terciario).

Con respecto a las falencias y debilidades, la opinión varía según el plan de estudios con el que haya cursado el entrevistado. Muchas de las debilidades marcadas por aquellos que cursaron con los planes más antiguos (“85”, “91”) fueron subsanadas con el plan “99”. ${ }^{2}$ Por otro lado, las falencias a las que se refieren los entrevistados en muchos casos no se deben tanto a los contenidos de las materias sino más bien a la forma en que esos contenidos fueron dados.

Otro aspecto señalado es la ausencia de articulación con la práctica. Advierten que es necesario una instancia de integración y de aplicación de ese conocimiento teórico. Porque, en ocasiones, no se sabe para qué sirve todo ese "bagaje teórico" o, en otras palabras, cómo utilizarlo, sobre todo en el caso de los sociólogos que se han dedicado a actividades relacionadas a la elaboración, ejecución y/o evaluación de proyectos, programas o políticas sociales:

A mi me parece que hay en Sociología, porque Trabajo Social no es asi, pero nosotros en Sociología, falta, por lo menos en mi época, faltaba mucha articulación, o sea, articulación con la práctica. O sea, vos salís con un bagaje teórico que no sabes para qué te sirve, en realidad. (socióloga, 51 años, recibida en 2011, sector estatal) 
Sin embargo, también reconocen que con los años de trayectoria laboral van asumiendo que la carrera de grado es "un marco general" o una plataforma de despegue y que luego cada uno va construyendo las alternativas y adquiriendo nuevas herramientas, destrezas y saberes dentro de los diferentes ámbitos laborales.

En cuanto a la formación metodológica, es importante tener en cuenta que las valoraciones sobre la misma van cambiando según las cohortes a las que pertenezcan los graduados. Mientras más antiguas son las cohortes, más quejas hay en relación a este aspecto. Como, por ejemplo, dentro del grupo de los graduados entrevistados que pertenecían a las primeras cohortes estudiadas, ${ }^{13}$ la formación estadística fue evaluada como muy básica e insuficiente. E inclusive consideraban que profesionales que provenían de otras disciplinas sociales y/o humanísticas, como economía o psicología, tenían una formación más sólida en estos temas. Además de la falta de manejo de software para procesamiento de datos estadísticos y datos cualitativos.

Luego, en el resto de las cohortes se señaló como una debilidad la falta de entrenamiento para poner en práctica las diferentes técnicas metodológicas aprendidas y la falta de análisis de casos concretos. Hubiera sido necesario, según estos graduados, más práctica en la elaboración de encuestas, operacionalización de variables, construcción de indicadores y luego en el procesamiento de datos. Finalmente, también fue señalado como una debilidad la ausencia de una oferta de materias metodológicas optativas que profundizaran lo que no se había alcanzado a ver en aquellas que eran parte obligatoria dentro del plan de estudios.

No obstante, hay quienes señalan que la formación metodológica fue suficiente como para tener una base y después profundizar en forma autodidacta. En algunos casos en donde los graduados han trabajado en equipos integrados por otros profesionales de las ciencias sociales, han percibido que la formación metodológica ha sido buena:

Entonces en lo que más fuerte que yo siento que me ha ayudado... la metodología como herramienta, pero en realidad el método. Nosotros... vos te das cuenta, cuando estamos en cualquier lugar, en educación y demás, cómo nos ha cuadrado el método de trabajo, el método de análisis. Eso para mi nos hace mucho ruido con todas las áreas de las ciencias sociales que no lo tienen, pero eso es bueno, porque quiere decir que estamos bien parados. (sociólogo, 43 años, recibido en 2004, sector estatal y docente en nivel terciario)

En este caso no estamos refiriendo a las cohortes que van del año 1983 al año 1990. Estas cohortes cursaron todas bajo el primer plan que se elaboró después de la reapertura de la carrera. 
Aquellos que están insertos en el ámbito público y/o ONGs consideran que los contenidos que les habían resultado más útiles para su posterior inserción fueron los brindados por las materias metodológicas (tanto técnicas cuantitativas como cualitativas). También los contenidos estudiados en la materia "Planificación y evaluación de proyectos" (sólo presente en plan 91 y plan 99) ya que aportaban algunas herramientas relacionadas con la gestión. Sin embargo, este único espacio curricular (con una carga horaria de sólo 80 horas), aislado dentro de un plan de estudios fuertemente orientado hacia el oficio académico, no alcanzaba por sí solo a cubrir las necesidades de una formación básica que preparara a los futuros sociólogos para realizar tareas de planificación y asesoramiento en el sector público a través del diseño, ejecución y/o evaluación de políticas sociales. De hecho, muchos de los entrevistados buscaron profundizar esos contenidos en sus estudios de posgrado.

La formación recibida les permitió contar con una mirada más abarcadora y más compleja de la realidad, ya que la carrera aporta esquemas de pensamiento alternativos, que rompen con el sentido común:

\footnotetext{
Yo siempre digo, la Facultad, para mi la Facultad te da esquemas de pensamiento. O sea, modos de enfrentarte a la realidad con un bagaje de esquemas conceptuales y de razonamiento que vos abordas la realidad desde ese lugar. (socióloga, 47 años, recibida en 1996, sector ONGs)
}

\section{CONTENIDOS O ACTIVIDADES QUE FALTARON}

Es importante advertir que quienes más destacaron la falta de herramientas prácticas son aquellos que a lo largo de su trayectoria se han dedicado frecuentemente a trabajos en territorio o ligados a actividades de ejecución de política pública. Dichos graduados observaban que faltaron herramientas de trabajo para el abordaje en comunidades, en el territorio y herramientas para saber cómo armar capacitaciones de profesores que dictan clases en la carrera. E 75\% del plantel docente de la Lic. en sociología se dedica en forma exclusiva a la actividad académica, es decir a la docencia universitaria y a la investigación (tanto dentro de la misma universidad como en organismos nacionales, por ej. CONICET) diversa índole, contar con herramientas para manejo de grupos, algunas herramientas de gestión, también algunos conceptos sobre armado de presupuestos, cómo calcular honorarios, capacitación en la búsqueda de fuentes de financiamiento, elaboración (incluyendo los costos financieros) y evaluación de proyectos, ${ }^{14}$ manejo de recursos humanos. Inclusive, alguno de ellos señaló que este tipo de contenidos o herramientas eran denostadas, es decir desacreditadas por los docentes de la carrera. ${ }^{15}$

La carrera no asumía la necesidad del debate sobre la futura inserción de los sociólogos en otros ámbitos que no fueran los académicos propiamente dichos (docencia e investigación). En otras palabras, los entrevistados percibían que los docentes no los 
acompañaron orientándolos en la futura búsqueda laboral antes de terminar el trayecto educativo. De hecho, una de las entrevistadas indicó que hubiera sido necesario contar con la presencia de tutores o profesores que los monitorearan durante toda la etapa del cursado y que les dieran más asesoramiento para vincularse al mercado laboral o ayuda para definir el rol del sociólogo.

Se ha señalado como debilidad la falta de prácticas profesionales dentro de los planes de estudio que se implementaron durante esos años. ${ }^{16}$ Ya que perciben que la carrera se encontraba desconectada de la realidad cotidiana, sobre todo para aquellos que trabajan en ámbitos no académicos como áreas del Estado, Organizaciones no Gubernamentales -ONGs- y/o ámbito privado. A su vez, esa falta de prácticas pre-profesionales le ha quitado visibilidad a la carrera y a la profesión dentro de los ámbitos extra-académicos por la inexistencia de convenios o de articulaciones con instituciones del ámbito público estatal y no estatal. Esta desvinculación de la carrera también genera a su vez que aquellos graduados que trabajan fuera del ámbito académico consideren que luego de egresar existe mucha dificultad para volver a vincularse con la Facultad y con la carrera.

Desde el punto de vista del oficio académico, advierten también que hubiera sido necesario tener más instancias de entrenamiento en la producción escrita (elaboración de ensayos, artículos, informes, etc.).

\section{CONFRONTACIÓN ENTRE LA SOCIALIZACIÓN UNIVERSITARIA Y LAS PRIMERAS EXPERIENCIAS EN EL MUNDO DEL TRABAJO}

Todo aquello que fue internalizado durante la etapa de estudiante universitario más las expectativas generadas por esos aprendizajes, son confrontados con la realidad a partir de las primeras experiencias laborales. Por ejemplo, una de las entrevistadas señalaba que después de haber "jurado", mientras estaba en la Facultad, que no iba a trabajar en el Estado, luego terminó considerando que éste era el espacio más potente para lograr transformaciones:

\begin{abstract}
[...] y es cierto que el espacio más potente para lograr transformaciones es el Estado porque llegás a mayor cantidad de gente y además a los sectores más vulnerables y es desde donde más podés hacer y entonces eso de "no quiero trabajar en el Estado" también entró en controversia. (socióloga, 45 años, recibida en 1998, sector estatal)
\end{abstract}

En efecto, muchos graduados señalan que, durante el periodo de formación en la facultad, se veía con desconfianza y escepticismo que actualmente está implementándose una reforma del plan de estudios que consiste en la apertura de dos nuevas cátedras: las "Prácticas sociales educativas" y las "Prácticas Profesionales", las cuales apuntan a solucionar la falta de preparación y de experiencia práctica de los alumnos para el ejercicio profesional. 
cualquier acción que pudiera llevarse a cabo desde el Estado destinada a los sectores más marginados.

La inserción profesional concreta ha implicado para el sociólogo una lucha interna o un esfuerzo por intentar mantener la "coherencia ideológica” entre la formación recibida en la carrera y la tarea cotidiana en su trabajo.

Como señalamos anteriormente, la gran mayoría de los sociólogos entrevistados son individuos que provienen de familias de sectores medios y han tenido su primera experiencia laboral una vez iniciados los estudios universitarios. ${ }^{17}$ En general, los primeros años de la facultad no trabajaron y la necesidad se planteó en los últimos años, por diversas razones (tener cierta independencia económica, para gastos personales, salidas, vacaciones, etc.).

La "primera inserción laboral” de aquellos que no habían trabajado antes de entrar a la universidad se dio, en todos los casos, durante los estudios universitarios y en la mayoría de éstos se trató de trabajos relacionados con la carrera como: realización de encuestas para organismos públicos como la Dirección de Estadísticas e Investigaciones Económicas ${ }^{18}$-DEIEy/o consultoras privadas, trabajos dentro de alguna ONG.

El trabajo como encuestador fue descripto por la mayoría como un "paso obligado" dentro de la etapa de estudiante universitario, pero que no implicó un trabajo estable. Sin embargo, también encontramos algunos casos de unos pocos que continuaron en ese camino y con el tiempo encararon tareas como supervisores de trabajo de campo, y

Sin embargo, existen algunos casos aislados en donde su primera experiencia laboral se registra a edades más tempranas, durante la adolescencia. En estos casos se trata de trabajos como empleados en un comercio trabajo por cuenta propia o en algún negocio familiar. cuidado de niños, dictado

de clases de apoyo particulares, changas. Provincia de Mendoza.

sonveniente aclarar que, para el caso de los estudiantes de sociología

estas oportunidades de

participar en pasantías organizadas por la Facultad fueron casos absolutamente aislados. Es decir, no existía una oferta permanente. ni se trataba de una práctica institucionalizada u obligatoria dentro del plan de estudios. luego coordinadores de operativos específicos.

Otra alternativa laboral durante los estudios universitarios fue ingresar, bajo la figura de una "pasantía” o "práctica profesional”, en algún programa o proyecto social, ya sea por algún convenio firmado entre la facultad ${ }^{19}$ y un organismo del Estado o por algún contacto personal (en la mayoría de los casos), para realizar tareas administrativas o de tipo técnicas como relevamientos, carga y/o sistematización de datos, atención al público o como tallerista/capacitador en el territorio.

De los que ingresaron a trabajar en alguna fundación u ONG, la mayoría lo hicieron en carácter de voluntarios, es decir, ad-honorem (salvo alguna oportunidad aislada en donde apareció la posibilidad de recibir algún pago por la captación de financiamiento), generalmente realizando tareas de capacitación, a cargo de talleres sobre diferentes temáticas (prevención de la salud, adicciones, violencia de género, microemprendimientos, entre otros).

Algunos trabajaron en actividades no relacionadas con la carrera, como la participación en algún emprendimiento o pequeño negocio familiar, como empleados administrativos en empresas privadas o dentro del ámbito público (en municipios, gobierno central o en la legislatura), trabajando como mozos, dando clases particulares de apoyo 
para alumnos de nivel medio, vendiendo tarjetas de crédito en bancos $\mathrm{u}$ otras formas autogestionadas de trabajo. Los trabajos temporarios fueron en general todos trabajos inestables, precarizados, no registrados. Inclusive algunas pasantías tardaban entre tres a seis meses para poder ser cobradas y otras directamente no se pudieron cobrar.

Cuando el objetivo de la inserción laboral era contar con una entrada fija y estable, entonces la búsqueda se orientaba más específicamente a la captación de empleos administrativos en empresas u organismos públicos o áreas de atención al cliente, así como actividades de capacitación en organismos públicos; como preceptores o como docentes en el nivel medio a través de convocatorias para cubrir cargos que habían quedado vacantes porque no se había presentado ningún profesor con título habilitante o por presentación de antecedentes o contacto personal en colegios de gestión privada. En estos casos, algunos lograron mejorar un poco su situación en cuanto a las condiciones de trabajo. Sin embargo, aquellos que consiguieron un trabajo registrado, en relación de dependencia y con bono de sueldo, fueron las excepciones a la regla.

Analizadas en perspectiva, estas experiencias, ya fueran más o menos relacionadas con la carrera, resultaron ser altamente relevantes y significativas en todas las trayectorias y por varias razones. En primer lugar, como entrenamiento para el trabajo (aprendizaje, adquisición de competencias), porque como estudiantes pusieron a prueba los conocimientos adquiridos, y además porque ayudaron a incrementar la red de contactos que luego sería clave para conseguir otros trabajos en el futuro.

\section{ESTRATEGIAS DE INSERCIÓN}

La vía de acceso a los trabajos fue, en la mayoría de los casos, a través de un contacto, ya sea familiares, amigos, compañeros de la facultad, compañeros de trabajo, por militancia o profesores, que aparte de su actividad docente tenían un trabajo dentro del ámbito público. Esto no significa que la experiencia previa y los antecedentes no contaran en absoluto. Sin embargo, los contactos jugaron un papel fundamental siempre. Lo cual indicaría una fuerte influencia del capital social que poseen los sujetos (BOURDIEU, 2000). Rara vez se trató de una convocatoria abierta o por presentación de antecedentes, salvo en los llamados para cubrir cargos docentes en los colegios secundarios de gestión pública: 
entrar a una cátedra. [...]. Si te hacías amigo de un docente que trabajaba en la Dirección de Estadísticas o en un Municipio, te... Así, asi, relaciones personales. Y a los que no teníamos esas relaciones personales, que no nos formamos desde ahi, yo creo que nos fue más difícil, nos fue más difícil porque fue parte de una búsqueda mucho más personal, mucho más individual y sentimos mucho la... Yo en lo personal y muchos colegas sentimos mucho la... Eh... a ver, cómo sería, la desatención de la Universidad, de la Facultad y de la Carrera. (sociólogo, 43 años, recibido en 2004, sector estatal y docente en nivel terciario)

Dentro del ámbito privado, si bien la red de contactos también fue imprescindible, se mencionó como una situación más frecuente la existencia de convocatorias abiertas o de búsquedas de personal a través de consultoras de recursos humanos, cuando la búsqueda estaba dirigida a trabajos dentro de una empresa. Distinto fue el caso, por ejemplo, de aquellos que se lanzaron a armar su propia consultora.

Aquellas actividades consideradas como "más acordes" o "más prestigiosas" para la salida laboral de un sociólogo, implican como estrategia de inserción recorrer una primera etapa de prácticas no rentadas, tales como ingresar a una cátedra como profesores adscriptos ad-honorem o integrar algún equipo de investigación, o trabajar como asistente de algún investigador. Lo cual, en algunos casos, termina convirtiéndose en una limitante para aquellos que no pueden disponer de ese tiempo ya que necesitan encontrar un trabajo con un ingreso estable.

\section{EL PROCESO DE "SOCIALIZACIÓN PROFESIONAL” Y LA INSERCIÓN LUEGO DE LA OBTENCIÓN DEL TÍTULO DE GRADO}

Como señalamos más arriba, para los graduados entrevistados el inicio de su trayectoria laboral se sitúa en la etapa de los estudios universitarios. Allí comienza un proceso de confrontación entre las ideas y percepciones que fueron internalizadas durante el proceso de socialización universitaria y aquellas primeras impresiones obtenidas a partir del ingreso al mundo laboral. Este proceso continúa y se

Se trata en general de contratos por una cierta cantidad de meses, bajo la figura de contratos de ocación o de servicios. En esas condiciones pueden permanecer varios años y según las circunstancias (políticas generalmente) logran el pase a planta. profundiza una vez obtenido el título.

Aquellos que logran insertarse en el ámbito público lo hacen en condiciones de precariedad con contratos temporales,$^{20}$ generalmente dentro de algún programa, cumpliendo tareas de tipo técnicas, como encargados de la recolección y sistematización de información pertinente a esa área o a cargo de capacitaciones y/o talleres. 
Algunas de las áreas, tanto dentro del estado provincial como municipal, en las cuales los sociólogos encontraron posibilidades de inserción fueron: educación, seguridad, género, salud, transporte, ambiente, empleo, economía social, niñez y adolescencia y otras áreas dentro de las políticas sociales. Sus funciones combinan diferentes tareas, como labores administrativas, recolección y procesamiento de datos, la ejecución de actividades que son parte de la implementación de algún programa, la elaboración de diagnósticos, tareas de capacitación, de vinculación con los municipios e instituciones de la comunidad en general, es decir, como cuadros técnicos. ${ }^{21}$ En algunos pocos casos nos encontramos con sociólogos que han estado como coordinadores generales de programas nacionales. Este tipo de trabajos incluyen múltiples tareas y responsabilidades: ${ }^{22}$

\begin{abstract}
Porque era el arranque del Programa y hubo que armarlo, desde armar el equipo técnico, de coordinador, armar los equipos técnicos en terreno, detectar a los técnicos. Y después hacer funcionar el Programa en el sentido de hacer una difusión del mismo, de llegada y que finalmente se pudiera llegar a los productores con... Se formaran los grupos y los productores pudieran recibir los créditos. No... Fue una tarea típica de gerencia pública, de gestión pública con distintos frentes porque tenías aspectos administrativos, contables, técnicos de promoción, de coordinación. Había que coordinar con la XX (Institución), con el gobierno de la provincia. [...]. Había como treinta técnicos en el terreno, que digamos era mi responsabilidad que cobraran, que presentaran informes. (sociólogo, 51 años, recibido en 1991, sector académico, antes director de programa nacional)
\end{abstract}

Es interesante ver, a modo de ejemplo, cómo algunos sociólogos han ido transitando distintas fases de su trayectoria dentro del área de estadística del gobierno provincial: el primer paso es haciendo encuestas, luego supervisando y capacitando a encuestadores, después estando a cargo o coordinando algún operativo de relevamiento de datos en particular. Paralelamente en el día a día, una vez que ya entraron y cumplen un horario fijo, comienzan a encargarse del procesamiento de los datos. Básicamente son generadores de datos y su principal usuario es el mismo gobierno, aunque no el único. Se ocupan de armar indicadores de diversa índole (económicos, sociales), elaborar informes y luego darlos a conocer a través de las publicaciones que tiene esta dependencia. Particularmente es más fácil poder visualizar ciertos recorridos estandarizados dentro de las trayectorias de sociólogos que han trabajado allí, dado que su estructura de funcionamiento no ha variado en muchos años y permanece igual a pesar de los cambios en la conducción política. Sin embargo, hay otras áreas que están más sujetas

21

A pesar de estar cumpliendo tareas de tipo técnicas su permanencia en esos lugares de trabajo está muy atada a los vaivenes políticos. Salvo en aquellos pocos casos que tienen un cargo de planta.

22

Además de las múltiples tareas es importante señalar que este tipo de puestos son percibidos como trabajos que incluyen una fuerte dimensión política por la cantidad de actores que están involucrados y por la cantidad de instituciones, estatales y no estatales, con las que es necesario establecer un vínculo. 
a los cambios en el diseño de las políticas públicas y los programas que se ejecutan en cada momento.

Los empleos en el ámbito público tienen jornadas con una duración de 6 a 8 horas diarias aproximadamente. Lo cual les permite a algunos de ellos tener otros trabajos, generalmente de carácter temporal o con una carga horaria mucho menor. Sobre este tema volveremos más adelante.

Las alternativas de inserción más comunes para los sociólogos dentro del ámbito privado son: ingresar a trabajar como empleados en una consultora o montar una consultora propia en forma individual o con algún socio. Las tareas van desde la selección de personal para alguna empresa, el armado de capacitaciones, la implementación y certificación de normas ISO o de acciones que apuntan a la responsabilidad social empresaria, hasta estudios de mercado y sondeos de opinión pública para campañas políticas. En estos casos los clientes pueden ser desde pymes, grandes empresas, organismos del Estado provincial o municipal, hasta funcionarios públicos. En la mayoría de los casos se trata de trabajos de autogestión, desde el armado de una red de contactos y potenciales clientes, la búsqueda de recursos, el armado de presupuestos, etc.

En los últimos años muchos sociólogos que trabajan en consultoras han comenzado a incursionar también en la realización de estudios de impacto ambiental.

Otra alternativa es ingresar a trabajar en relación de dependencia en una empresa dentro del área de Recursos Humanos. Aquí las tareas pueden ser: la capacitación de personal, la realización de diagnósticos institucionales y la comunicación institucional. También trabajan en la elaboración y ejecución de proyectos de desarrollo organizacional ligados al mejoramiento de la calidad de servicios.

La inserción en Fundaciones y ONGs es menos común si lo pensamos en términos de trabajos rentados, sobre todo cuando se trata de sujetos que ya se recibieron, pero existen algunos casos. Dentro de estas instituciones los sociólogos se encargan básicamente de armar proyectos y buscar organismos para su financiamiento. De hecho, este mecanismo se convierte frecuentemente en el único camino para poder permanecer trabajando dentro de una ONG sin tener que caer en un trabajo voluntario. En general se trata de trabajos no registrados o trabajando como consultor independiente y facturando como monotributista.

El ejercicio de la docencia en el nivel secundario y terciario es también otra forma muy generalizada de inserción laboral entre los sociólogos. Hay quienes se dedican tiempo completo a esta actividad no solo dando clases frente al curso, sino además trabajando en tareas de gestión y coordinación académica y/o en la dirección de carreras (en el caso de los Institutos terciarios). Mientras que otros sociólogos dan 
solo algunas horas de clase como complemento de otro trabajo que representa su principal ingreso. Existe también el caso de aquellos que, sin tener en sus planes el dedicarse a la docencia (sobre todo a la docencia en el nivel medio) toman algunas horas, ya sea porque están dando sus primeros pasos como graduados o porque el contexto socioeconómico los ha obligado buscar ciertas estrategias de sobrevivencia. Para estos últimos, la docencia es tomada como una "ocupación refugio".

No podemos dejar de mencionar aquellos casos en donde se combinan diferentes actividades: en general un trabajo estable, como por ejemplo un cargo en el ámbito público (como señalamos más arriba) y otros trabajos que implican menos horas de dedicación, como por ejemplo procesamiento de datos para terceros, estudios de impacto ambiental, el dictado de algunas horas de clase ya sea a nivel secundario, terciario y en algunos pocos casos, a nivel universitario. También se combinan con la participación esporádica en la elaboración y/o ejecución (o realización de consultorías para) proyectos de diversa índole. Estos proyectos pueden tener una duración entre 6 meses y 2 años, y frecuentemente crean una especie de "encadenamiento" unos con otros. Es decir, la participación en un proyecto genera los contactos con diversos actores y la experiencia y los canales de información necesarios para acceder a las convocatorias de nuevos proyectos.

Los contactos personales siguen siendo la vía de acceso principal. Si no es directamente para acceder al puesto, al menos es "para enterarse" dónde ha surgido una demanda que pueda ser acorde a los intereses y/o al perfil de ese graduado. Esto se debe a que los canales de acceso a la información son, en la mayoría de los casos, cerrados. Sin embargo, existen otras estrategias menos utilizadas por los sociólogos dentro de ciertos ámbitos como el público, pero que pueden ser más efectivas cuando el objetivo es ingresar en el ámbito privado. Nos referimos a enviar currículos a las consultoras privadas que se encargan de seleccionar personal para empresas.

Lógicamente, una vez establecido el contacto, los antecedentes pueden ser decisivos a la hora de ser seleccionado para cubrir un puesto determinado, pero no siempre es prioritario este criterio.

La excepción a estas situaciones se da en el acceso a los cargos docentes en el nivel secundario, cuyas convocatorias son abiertas y publicadas en los diarios de mayor tirada y el criterio de selección es a través del bono de puntaje que otorga la Dirección General de Escuelas -DGE.

Con respecto al trabajo actual, al momento de realizar las entrevistas todos los sociólogos menos dos estaban trabajando en ocupaciones relacionadas con su profesión; ${ }^{23}$ sin embargo, por limitaciones de espacio, no podremos desarrollar este tema para cada una de las trayectorias. Sólo mencionaremos que trabajamos sobre los

23

Cuando hablamos de trabajos relacionados con la profesión", nos referimos a todas aquellas ocupaciones que involucren una o más actividades contempladas dentro de las incumbencias que figuran tanto en el plan de estudios como aquellas que incluye la Resolución n¹818/86 del Ministerio de Educación de la Nación. 
siguientes aspectos que caracterizan el trabajo actual: Características generales, aspectos que más les atrae, el motivo de la elección, los aspectos negativos, la percepción sobre los conocimientos que aplica en la práctica, las primeras impresiones, reflexiones sobre sus características, la vía de acceso, el trabajo en equipo y los aprendizajes en el empleo. Y otros aspectos que hacen a la reflexión de los entrevistados sobre sus propias trayectorias: los hitos más importantes y la mirada global que tienen sobre la misma. Otros elementos que surgieron de las entrevistas son: las percepciones sobre las características del empleo en el sector privado, en el sector público, en las ONGs y en el sistema académico; la relación entre el oficio del sociólogo y la dimensión política.

Nos detendremos solo en dos aspectos: la percepción sobre el sueldo y el tipo de relación laboral.

Dentro del ámbito público, los sueldos pueden variar mucho, ya que existen diferentes escalafones. Por ejemplo, en el sistema penal los sueldos son más altos que los de la administración central (poder ejecutivo). Y también existen diferencias con respecto a los sueldos de la administración nacional.

En general, los sociólogos entrevistados no están conforme con lo que ganan. Advierten, además, que existen ciertas diferencias con respecto a los sueldos si se comparan con otros profesionales que realizan tareas o tienen responsabilidades similares, por ejemplo, los trabajadores sociales o los abogados. La situación mejora considerablemente en los cargos de funcionario público, pero entre los sociólogos estos constituyen casos muy aislados.

En el caso de las mujeres con hijos muchas veces el contar con un "buen sueldo" es resignado en pos de otros aspectos como la flexibilidad horaria, lo cual les permite encargarse personalmente de la crianza y de los cuidados de los mismos.

En relación al trabajo desarrollado en las ONGs, en estos espacios se advierte la dificultad para que la tarea del sociólogo sea reconocida económicamente.

Con respecto a la relación laboral, muchos de ellos, a pesar de llevar más de tres años en sus trabajos, no han conseguido ingresar a planta. ${ }^{24}$ Es decir, que están con contratos temporales o facturando como monotributistas, inclusive durante los primeros años figuraban como pasantes de la universidad. Como por ejemplo, aquellos que trabajan en la Administración Central o dentro de la Universidad en cargos no docentes (estos últimos sufren más fuertemente los avatares producidos por los cambios de gestión que vulneran la estabilidad en sus puestos).

Distinta es la situación, por ejemplo, de aquellos que tienen un cargo docente efectivo en la universidad. En este caso, y más allá de otros aspectos negativos, se valora mucho la autonomía, el respaldo y 
la estabilidad que otorga el poder acceder (vía concurso) a un cargo de este tipo.

Aquellos que se dedican a los trabajos de consultoría lo hacen por cuenta propia, en calidad de trabajadores independientes, en forma autogestionada y facturan como monotributistas.

Dentro del ámbito de la docencia a nivel secundario y terciario, la relación laboral en general está registrada, cuentan con un bono de sueldo que incluye todos los aportes y cargas sociales. No así en el caso de los colegios privados, en donde la relación laboral está más precarizada e inestable.

\section{¿QUÉ IMPLICA "SER SOCIÓLOGO” PARA LOS SOCIÓLOGOS?}

Durante el desarrollo de sus trayectorias los sociólogos van construyendo determinadas representaciones de sí mismos, del colectivo del cual forman parte y sobre la relación que establecen en el ámbito laboral con otras profesiones. Dichas representaciones se van configurando a través de las distintas etapas del proceso de socialización mencionadas anteriormente. Aunque estas representaciones no son establecidas de una vez y para siempre, influyen fuertemente en la forma en que los sujetos transitan sus trayectorias laborales y en la manera en que toman decisiones y elaboran proyectos a futuro. Y aunque los sociólogos son conscientes de que el fundamento de esas representaciones se origina en gran medida durante el mismo proceso de formación, estas ideas no dejan de tener una fuerte influencia en su accionar.

Como señalábamos al principio, aquellos que no están insertos en el campo académico perciben que existen concepciones impartidas desde la misma carrera sobre qué trabajos están más acordes con el perfil profesional del sociólogo y que crean a su vez una frontera que deja al margen otro tipo de trabajos que no son ni la investigación ni la docencia universitaria. Se advierte, por ejemplo, que algunos profesores dejan entrever cierta descalificación por el ejercicio de la docencia en el nivel medio como una inserción poco acorde con el perfil del sociólogo. Más bien se lo considera como una estrategia de sobrevivencia o, como señalamos anteriormente, una "ocupación refugio".

Otras veces se piensa al sociólogo en contraposición con el trabajador social y de esta manera se debilita la posibilidad de ejercer un rol de intervención desde la sociología.

De hecho, hay quienes perciben que el perfil profesional que otorga la carrera es "demasiado general, muy teórico y con muy poca aplicación práctica”. Y que esta formación entonces sirve más si uno quiere ser docente o investigador, pero no para trabajar en instituciones. Para los propios entrevistados, los “sociólogos típicos” son considerados 
como poco pragmáticos, con ciertas dificultades para arribar a resultados concretos. Son vistos en ocasiones como "individualistas" y generan cierto rechazo en los trabajos interdisciplinarios por su excesivo teoricismo, por la “crítica exagerada”, y cierta incapacidad para proponer alternativas concretas de acción. Hay quienes señalaban que el sociólogo tiene más capacidad para generar diagnósticos que para tomar decisiones, es de "estar atrás de las bambalinas".

Dentro del ámbito público se advierte, por ejemplo, que la dirección de ciertos procesos está en manos de otros profesionales como los abogados, que son quienes generalmente ocupan cargos directivos, cargos jerárquicos. Son los “que tienen la firma”. En otras palabras, los abogados son los que principalmente detentan el poder en las diversas áreas del Estado. Existe efectivamente "una subordinación a lo jurídico" advierten algunos. También los contadores tienen a su cargo la toma de decisiones:

[...] yo lo que veo es que se manejan todas las decisiones que se toman, primero se analizan los criterios económicos a ver si cuadran los números y si cuadran los números, ahi se hace un análisis legal para ver si encuadra dentro de la ley y si está todo ok se aprueba, se hace. Pero esos son los criterios que yo veo que se toman, nada más económicos y legales. (socióloga, 36 años, recibida en 2011, sector estatal)

\section{PERCEPCIÓN SOBRE LA SITUACIÓN ACTUAL EN EL MERCADO LABORAL}

En general existe una percepción poco alentadora sobre el panorama laboral para los sociólogos, sobre todo para los graduados recientes. Sin embargo, esta es una situación que comparten con la gran parte de los profesionales de otras disciplinas de las ciencias sociales que se encuentran en esa misma etapa de sus trayectorias.

En cuanto a las posibilidades de inserción que ofrece el ámbito académico, hay quienes señalan que, si bien se pueden encontrar muchos sociólogos insertos en equipos de investigación, estos lo hacen ad honorem, teniendo que dedicarle un horario extra fuera de la jornada laboral.

A su vez, algunos de los sectores no académicos en donde potencialmente pueden ser requeridos los sociólogos, siguen sin desarrollarse plenamente, como es el caso del campo del análisis estadístico en el ámbito local.

Dentro del ámbito público, las condiciones laborales son muy vulnerables a la variable política, aun cuando se trate de cargos técnicos. Otro inconveniente al que deben enfrentarse es que, en la mayoría de 
las Instituciones que componen el Estado, "no tienen ni idea de lo que es la sociología” o a lo que se dedican los sociólogos.

Dentro del ámbito de la docencia en el nivel medio, los sociólogos deben afrontar una fuerte disputa con otros profesionales (profesores de historia, de filosofía, psicólogos, licenciados en ciencias de la educación, psicopedagogos, trabajadores sociales) por la distribución de los espacios curriculares de las ciencias sociales (es decir, quién tiene la prioridad para tomar el cargo en cada caso). Sobre todo, cuando la oferta para esos espacios curriculares se achica como está sucediendo actualmente a nivel local.

Muchas veces las posibilidades de inserción laboral para los graduados están condicionadas a la eventual ejecución de programas y/o proyectos con una duración acotada, que implica el tener que estar "saltando" de un proyecto al otro sin tener la posibilidad de lograr estabilidad. Y sumado a esto vuelve a aparecer el limitante de los canales de información sobre las convocatorias y ofertas laborales que siempre son cerrados.

Para muchos el rol del sociólogo no está reconocido socialmente, como sí sucede con otras profesiones, como por ejemplo el Trabajador Social, que ha logrado mejor inserción en el sector público. Los entrevistados señalan que tienen otro régimen laboral gracias a que cuentan con el apoyo de un colegio y una matriculación dentro del Estado.

A su vez, la superposición de incumbencias con otras profesiones genera conflictos en todos los ámbitos. En el ámbito público, con el Trabajador Social, el Licenciado en Ciencias Políticas y con el Psicólogo Social en temas vinculados al abordaje de las problemáticas sociales. Y en el ámbito privado con los publicistas, los licenciados en marketing, los psicólogos, los licenciados en recursos humanos y los administradores de empresas. De hecho, aquellos que está insertos en el ámbito privado también señalan que los sociólogos están perdiendo o desaprovechando espacios en donde otras carreras están tomando terreno, como es el caso de los psicólogos. Es decir que existen muchas alternativas de inserción laboral para los sociólogos, pero que se desconocen o se descartan por no ser acordes al ideal de sociólogo internalizado durante la carrera. Algunos señalan que la situación es diferente en conglomerados urbanos más grandes en donde, por ejemplo, la demanda de sociólogos para trabajar en empresas privadas, en consultoras o en agencias de publicidad es mucho mayor.

Mientras tanto, en el espacio conformado por las ONGs la percepción es que la actividad de los sociólogos es prácticamente inexistente, cuando en realidad estas instituciones tienen gran potencial como alternativas laborales. Sin embargo, el punto débil de las ONGs es que se trata de un proceso de "autogestión pura”. Es decir, "nadie 
va a pedir un sociólogo o va poner un aviso en el diario". Se señala que, en todo caso, es el sociólogo quien se acerca a las organizaciones para ofrecer su trabajo y es quien "inventa” o "crea” sus funciones o sus tareas dentro de estas instituciones. Y que la única manera de asegurar un ingreso por esas tareas es a través de "la captación de recursos vía la presentación de proyectos de organismos que potencialmente pueden otorgar financiamiento.

En definitiva, para el colectivo de profesionales de la sociología, “cada uno va construyendo su camino" y no existe otra alternativa. Se repite el tema de la "autogestión” de la inserción laboral y se lo ve en este caso como un factor limitante, como una debilidad sobre todo por la falta de una acción colectiva.

Se advierte sobre la ausencia de un diagnóstico de la situación laboral de los sociólogos y, además, que la carrera no tiene visibilidad, que es necesario realizar un mapeo y a partir de eso intentar revertir esta situación. Esto se ve como un paso fundamental si se quiere lograr cierta identidad profesional. Se señala como un problema o un obstáculo para la organización como colectivo, el rechazo que tienen los sociólogos a la idea de la organización corporativa.

\section{ALGUNAS CONSIDERACIONES FINALES}

Estas líneas constituyen una primera aproximación al objeto de análisis. Sin embargo, podemos constatar hasta aquí que efectivamente existe cierto grado de desarticulación entre el perfil profesional propuesto por el plan de estudios, la formación recibida y las oportunidades de inserción laboral para los sociólogos en el mercado de trabajo. Y que, si bien las transformaciones sufridas por la sociedad a partir de la década de los noventa, junto con la racionalización estatal y de la empresa privada impulsaron la expansión y diversificación de los espacios de inserción de los sociólogos, este proceso ha estado lejos de desarrollarse sin algunas dificultades. En primer lugar, los graduados consideran que no tienen elementos como para construir un puente entre lo que aprendieron durante la carrera y los potenciales espacios de inserción laboral, sobre todo durante la etapa de la primera inserción laboral. Se encuentran desorientados al no saber cómo diseñar estrategias que les permitan conseguir algún trabajo acorde con lo que estudiaron. A su vez, descubren que fuera de la facultad existe un gran desconocimiento sobre el oficio del sociólogo. Esto traería aparejado una baja o inexistente demanda específica de dichos profesionales en los ámbitos no académicos.

Sumado a esto, los sociólogos deben competir muchas veces con profesionales provenientes de otras disciplinas que han desarrollado más sólidamente mecanismos de defensa de sus incumbencias a través 
de organizaciones corporativas, como los colegios profesionales, y de leyes que dictaminan el ejercicio profesional de las mismas.

Si bien podemos encontrar algunos de ellos en diferentes áreas del ámbito estatal, una gran mayoría se encuentra cumpliendo tareas de tipo técnicas (procesamiento estadístico, capacitaciones, elaboración de informes). Y si tenemos en cuenta las características del perfil profesional incluido en los planes de estudio, sus graduados deberían tener una presencia mucho más consolidada en la organización y/o coordinación de los procesos de elaboración de las políticas públicas. Por el contrario, actualmente estas decisiones están, la mayoría de las veces, en manos de abogados o economistas, fenómeno que ha sido ampliamente investigado (DEZALAY; GARTH, 2002; NEIBURG; PLOTKIN, 2004, entre otros).

Consideramos que un plan de estudios no puede abarcar absolutamente todo, ni puede satisfacer las demandas de formación tanto teóricas como metodológicas en su totalidad. Tampoco es factible que vaya mutando permanentemente en función de los cambios en el mercado de trabajo. Es necesario evaluar hasta qué punto todos aquellos conocimientos más específicos, que los graduados le reclaman a la carrera, no debieran adquirirse en una etapa posterior de formación que convendría transitar luego de un cierto período de entrenamiento y experiencia en el campo laboral. Sin embargo, una carrera no puede ser indiferente a las problemáticas laborales que viven sus graduados cuando intentan insertarse en el mercado de trabajo. No obstante, corresponde señalar que no consideramos a la formación de grado universitaria como la clave principal para una inserción laboral "exitosa”, ya que queda claro que existen otros factores que influyen fuertemente en el tipo de inserción, como el capital social y cultural de los sujetos. ${ }^{25}$

Entendemos que como carrera tenemos mucho que mejorar para poder dar una respuesta más adecuada frente a la dinámica que plantea hoy el mundo del trabajo para los sociólogos, sobre todo para el caso de los que recién están iniciando su carrera profesional. Sin embargo, el lograr avanzar o no dentro del proceso de profesionalización de una disciplina no es exclusivamente responsabilidad de aquellas instituciones dedicadas a la formación profesional, sino también de aquellos que constituyen el colectivo profesional, es decir, los mismos graduados que, habiendo iniciado su trayectoria laboral, tienen la capacidad de contribuir al fortalecimiento de ese proceso.

\section{REFERENCIAS}

BELTRÁN, Gastón. Formación profesional y producción intelectual en tiempos de cambio político Las carreras de Sociología y Economía de la Universidad de Buenos Aires durante los años noventa. En: BUCHBINDER, P. Espacio público y privatización del conocimiento. Estudios sobre políticas universitarias en América Latina. Buenos Aires: CLACSO, 2005. p. 463-512. en la oposición entre las estructuras sociales y las estrategias individuales, ni entre los aspectos objetivos y subjetivos a la hora de explicar las trayectorias. 
BELTRÁN, Gastón. Las Ciencias Sociales y el surgimiento de un mercado del saber experto. Las bifurcaciones de la sociología argentina en el final del Siglo XX. En: RUBINICH, L.; BELTRÁN, G. (Ed.) ¿Qué hacen los Sociólogos? Buenos Aires: Aurelia Rivera, 2010. p. 49-72.

BLANCO, Alejandro. Razón y modernidad: Gino Germani y la sociología en la Argentina. Buenos Aires: Siglo XXI, 2006.

BLOIS, Juan Pedro. Sociología y mundo del trabajo. Las trayectorias laborales de los sociólogos de la UBA desde la restauración de la democracia. Sociedad, Revista de la Facultad de Ciencias Sociales de la UBA, Buenos Aires, n. 29/30, p. 197-210, 2011.

BLOIS, Juan Pedro. Mundo universitario vs. mundo laboral. El caso de los jóvenes sociólogos de la Universidad de Buenos Aires. Aposta, Revista de Ciencias Sociales, Madrid, n. 52, p. 1-27, Enero/Marzo 2012. Disponible en: http://www.apostadigital.com/revistav3/hemeroteca/pblois. pdf. Aceso en: jun. 2017.

BLOIS, Juan Pedro. El mercado de trabajo de los sociólogos en Argentina desde la vuelta de la democracia. El caso de los graduados de la UBA. Trabajo y Sociedad, Santiago del Estero, n. 22, p. 103-122, 2014.

BONALDI, Pablo; BLOIS, Juan Pedro. ¿Intelectuales, expertos o académicos? La socialización universitaria de los sociólogos en la Universidad de Buenos Aires desde la vuelta a la democracia. Revista Virajes, Caldas, CO, v. 16, n. 1, p. 65-88, enero/jun. 2014.

BOURDIEU, Pierre. Las formas del capital. Capital Económico, capital cultural y capital social. En: BOURDIEU, P. Poder, derecho y clases sociales. Barcelona: Desclée, 2000. p. 131-164.

BOURDIEU, Pierre; PASSERON, Jean-Claude; CHAMBOREDON, Jean-Claude. El oficio del sociólogo. Buenos Aires: Siglo XXI, 2002.

DELICH, Francisco. Crítica y autocrítica de la razón extraviada. 25 años de sociología. Caracas: El Cid, 1977.

DE VENANZI, Augusto. La sociología de las profesiones y la sociología como profesión: un estudio del papel ocupacional del sociólogo en la Administración Pública Nacional. Caracas: Universidad Central de Venezuela, CDCH, 1990.

DEZALAY, Yves; GARTH, Bryant G. La internacionalización de las luchas por el poder: la competencia entre abogados y economistas por transformar los Estados Latinoamericanos. Bogotá: Universidad Nacional de Colombia; ILSA, 2002.

DIEZ, M. Agustina. El dependentismo en la Argentina: una historia de los claroscuros del campo académico entre 1966 y 1976. Tesis (Doctoral) - Universidad Nacional de Cuyo, 2009.

Disponible en: <http://bdigital.uncu.edu.ar/fichas.php?idobjeto=3496>. Acceso en: jun. 2017.

DIEZ, M. Agustina. Career-building in a highly politized period: argentine social scientists in the 60s. In: BEIGEL, F. (Ed.). The politics of academic autonomy in Latin America. Public intellectuals and the sociology of knowledge. England: Ashgate, 2012. p. 187-203.

FICARDI, Marcela. Transmisión y oficio de la Sociología en Mendoza. Formación del campo profesional. Tesis (Maestría) - FLACSO, Buenos Aires, 2013.

GONZALEZ, Horacio. Historia crítica de la sociología. Buenos Aires: Colihue, 2000.

LONGO, M. Eugenia. Trayectorias laborales de jóvenes en Argentina. Tesis (Doctoral) -Universidad de Buenos Aires; Université de Provence. Aix-Marseille I, Buenos Aires, París, 2011.

MUÑIZ TERRA, Leticia. Carreras y trayectorias laborales: una revisión crítica de las principales aproximaciones teórico-metodológicas para su abordaje. Revista latinoamericana de metodología de las Ciencias Sociales, v. 2, n. 1, p. 36-65, primer semestre 2012.

NEIBURG, Federico; PLOTKIN, Mariano. Intelectuales y Expertos. Buenos Aires: Paidós, 2004.

NOÉ, Alberto. Utopía y desencanto. Creación e institucionalización de la Carrera de Sociología de la Universidad de Buenos Aires: 1955-1966. Buenos Aires: Miño y Dávila, 2005.

PANAIA, Marta. Inserción de jóvenes en el mercado de trabajo. Buenos Aires: La Colmena, 2009. 
PANAIA, Marta. Una revisión de la sociología de las profesiones desde la teoría crítica del trabajo en la Argentina. Santiago de Chile: Naciones Unidas, 2008. 42 p. (CEPAL. Documentos de proyectos). Disponible en: http://repositorio.cepal.org/bitstream/handle/11362/3619/S2008114 es.pdf?sequence=1\&isAllowed=y. Acceso en: oct. 2016.

PEREYRA, Diego. Cincuenta Años de la Carrera de Sociología de la UBA. Algunas notas contracelebratorias para repensar la historia de la sociología en Argentina. Revista Argentina de Sociología, Buenos Aires, año V, n. 9, p. 153-159, 2007.

PEREYRA, Diego et al. Atención, sociólogos trabajando. Desafíos de la inserción profesional de los primeros sociólogos y sociólogas en Argentina (1961-1985). Política \& Sociedade, Revista de Sociología Política, v. 14, n. 31, p. 227, 2015. DOI: http://dx.doi.org/10.5007/21757984.2015v14n31p227. Acceso en: oct. 2016.

RUBINICH, Lucas. Los sociólogos intelectuales: cuatro notas sobre la sociología de los años sesenta. Apuntes de Investigación del CECYP, Buenos Aires, n. 4, p. 31-55, 1999.

RUBINICH, Lucas; BELTRÁN, Gastón (Ed.). ¿Qué hacen los Sociólogos? Buenos Aires: Aurelia Rivera, 2010.

TESTA, Julio. Situación ocupacional e inserción profesional de egresados recientes de la Facultad de Ciencias Sociales. Estudios n. 1: Graduados de Relaciones del Trabajo 1987/1990. Buenos Aires: FCS/CBC, 1996.

WRIGHT MILLS, C. La imaginación sociológica. Oxford: Oxford University, 1959.

MARÍA AGUSTINA DIEZ

Consejo Nacional de Investigaciones Científicas y Tecnológicas -CONICET- y Universidad Nacional de Cuyo -UNCuyo-, Mendoza, Argentina agustinadiez@mendoza-conicet.gob.ar 